\title{
What happens to the bone flap? Long-term outcome after reimplantation of cryoconserved bone flaps in a consecutive series of 92 patients
}

\author{
Lennart Henning Stieglitz • Christian Fung • \\ Michael Murek • Jens Fichtner • Andreas Raabe • \\ Jürgen Beck
}

Received: 1 October 2014 / Accepted: 5 December 2014 /Published online: 24 December 2014

(C) Springer-Verlag Wien 2014

\begin{abstract}
Background Reimplantation of cryoconserved autologous bone flaps is a standard procedure after decompressive craniotomies. Aseptic necrosis and resorption are the most frequent complications of this procedure. At present there is no consensus regarding the definition of the relevant extent and indication for surgical revision. The objective of this retrospective analysis was to identify the incidence of bone flap resorption and the optimal duration of follow-up.

Methods Between February 2009 and March 2012, 100 cryoconserved autologous bone flaps were reimplanted at the Department of Neurosurgery, Inselspital Bern. Three patients were not available for follow-up, and five patients died before follow-up. All patients underwent follow-up at 6 weeks and a second follow-up more than 12 months postoperatively. A clinical and CT-based score was developed for judgment of relevance and decision making for surgical revision.

Results Mean follow-up period was 21.6 months postoperatively (range: 12 to 47 months); $48.9 \%$ (45/92) of patients showed no signs of bone flap resorption, $20.7 \%$ (19/92) showed minor resorption with no need for surgical revision, and $30.4 \%$ (28/92) showed major resorption (in $4 \%$ of these the bone flap was unstable or collapsed).

Conclusions Aseptic necrosis and resorption of reimplanted autologous bone flaps occurred more frequently in our series
\end{abstract}

This work has not been previously presented in part or in whole, and it has not been concurrently submitted elsewhere.

\section{H. Stieglitz $(\bowtie)$}

Department of Neurosurgery, Zurich University Hospital,

8091 Zurich, Switzerland

e-mail: lennart.stieglitz@usz.ch

C. Fung $\cdot$ M. Murek $\cdot$ J. Fichtner $\cdot$ A. Raabe $\cdot$ J. Beck Department of Neurosurgery, Bern University Hospital, Bern, Switzerland of patients than in most reports in the literature. Most cases were identified between 6 and 12 months postoperatively. Clinical observation or CT scans of patients with autologous bone flaps are recommended for at least 12 months. Patientspecific implants may be preferable to autologous bone flaps.

Keywords Aseptic bone flap necrosis · Bone flap · Bone flap resorption $\cdot$ Hemicraniectomy

\section{Introduction}

Reconstruction of skull defects is currently a standard procedure in neurosurgery and maxillofacial surgery. Reconstruction techniques date back to ancient times; Artico et al. provide a good overview of the historical development [3]. More recently, artificial materials have been developed that provide very good replacements, but are quite costly. For this reason, and following the paradigm that autologous material should always be preferred over artificial replacements, reimplantation of a bone flap is usually the first choice to close a bone defect [10]. The material provides perfect biocompatibility, shape and fusion with the surrounding bone [12]. For preservation of an explanted flap until the delayed reimplantation, the material is either implanted into a subcutaneous pouch $[2,13]$ or cryoconserved [1, 14]. However, several major complications are related to reimplantation of autologous bone, e.g., epidural and subgaleal fluid collections and bleedings, infections and resorption (aseptic necrosis) of the bone flap. 


\section{Material and methods}

Patients

Between February 2009 and March 2012 we reimplanted 100 cryoconserved autologous bone flaps at the Inselspital in Bern, Switzerland. Five patients died during follow-up (no perioperative deaths), and three patients were not available for follow-up. Of the 92 patients included in the present retrospective study, 60 were male and 32 female. The mean age was 46.2 years [standard deviation (SD) 18.0]. Pathologies leading to craniectomy were 39 cases of head trauma, 18 cerebrovascular insults, 17 intracerebral hemorrhages, 14 subarachnoidal hemorrhages and 4 cases of infectious disease leading to malignant brain swelling. The mean follow-up time was 21.6 months (SD 1.5).

This retrospective analysis was approved by the local ethics committee (Kantonale Ethikkommission Bern, Switzerland, approval no. 155124, 20.02.2013).

Technique of bone flap preservation and reimplantation

After explantation of the bone flap and the intraoperative decision for a delayed reimplantation, the bone was freed from adherent soft tissue residuals and washed carefully with $0.9 \%$ $\mathrm{NaCl}$ with $320 \mathrm{mg} / \mathrm{l}$ gentamycin (provided by the hospital pharmacy). Afterwards the bone flap was packed and sealed in a sterile plastic bag, which was stored at $-80{ }^{\circ} \mathrm{C}$.

For reimplantation the bone was taken directly from the freezer and thawed in the operating room in sterile $0.9 \% \mathrm{NaCl}$ solution with $320 \mathrm{mg} / \mathrm{l}$ gentamycin at $37^{\circ} \mathrm{C}$. After preparation of the surrounding skull the flap was reimplanted and fixed using titanium plates and screws (Low Profile Neuro 0.6-mm self-drilling screws, 4-5 mm length, 400.83×) and Double-Yor burr-hole-cover plates, $0.5 \mathrm{~mm}$ thick (product nos. 421.516 and 421.528; Synthes, Oberdorf, Switzerland).

\section{Follow-up}

All patients received a CT scan on the first postoperative day to detect possible hygroma, epidural or subdural hematoma, intraparenchymatous hemorrhage on the ipsi- or contralateral side, or hydrocephalus. Most patients were discharged to continue stationary rehabilitation after 3 to 5 days. After 6 weeks all patients received a clinical checkup in the outpatient clinic to identify cases of wound healing disturbance or delayed malresorptive hydrocephalus. A patient in questionable condition received an additional CT scan. All patients were scheduled for a second clinical follow-up after at least 12 months to rule out delayed resorption of the bone flap. In case of questionable resorption the patients received another CT scan at the 12-month visit.
Judgment of bone resorption

At the 12-month follow-up visit (or later), after reimplantation of the bone flap the patients were asked about headaches, palpable holes in the reimplanted bone or gaps around its margins. They were also asked about palpable instability and changes in appearance. Afterwards, the bone flap was examined by a physician through careful palpitation. In case of a stable, cosmetically good and clearly non-resorbed bone flap, we dispensed with an additional CT scan to avoid unnecessary exposure to ionizing radiation. For identified holes, gaps and instability, we assigned scores as described in Table 1.

Depending on the score, we derived indications for further follow-up and surgical revision in accordance with Table 2. As instability cannot be accepted in a reimplanted bone flap, we decided to assign 3 additional points for this condition. Thus, instability alone was considered indicative for surgical revision.

\section{Results}

Surgical outcome and complications

Mean dimensions of the craniectomies approximated in the 2D-lateral CT-scout view were $112 \mathrm{~cm}^{2}$ (SD 22). Complications, including bone flap resorptions, are listed in Table 3. Reimplantations of bone flaps were performed an average of 2.6 months after explantation (SD 1.5 months; range 0.3 to 7.4 months).

Table 1 Bone flap resorption score

\begin{tabular}{lllc}
\hline & $\begin{array}{l}\text { CT scan } \\
\text { (if available) }\end{array}$ & $\begin{array}{l}\text { Visible/palpable } \\
\text { (no CT scan available) }\end{array}$ & Points \\
\hline $\begin{array}{l}\text { Gaps or holes } \\
\text { (not }\end{array}$ & None & Not palpable or visible & 0 \\
temporobasal) & $<2 \mathrm{~cm}$ & & 1 \\
& $\geq 2,<3 \mathrm{~cm}$ & & 2 \\
& $\geq 3 \mathrm{~cm}$ & Palpable/visible & 3 \\
Bone thickness & $\geq 1 \mathrm{~mm}$ & Cannot be judged & 0 \\
Bone flap stability, & $<$ Bone & Stable & +1 \\
dislocation, & thickness & Visible or palpable & 0 \\
collapse & $\geq$ Bone & instability/dislocation/ & +3 \\
& thickness & collapse &
\end{tabular}

If a computed tomography (CT) scan was available, the resorption of the bone flap was judged based on the appearance of gaps and holes, after reduction of bone thickness and stability. If no CT scan was available, bone thickness could not be determined. For a palpable gap or hole in the flap a maximum of 3 points was assigned. In case of palpable instability of the flap or dislocation in the CT scan, 3 additional points were assigned 
Table 2 Indications derived from the bone flap resorption scores

\begin{tabular}{ll}
\hline Bone flap resorption score & Indication \\
\hline 0 Points (no resorption) & Clinical follow-up \\
1-2 Points (minor resorption) & Follow-up with CT scan after 6 months \\
$\geq 3$ Points (major resorption) & $\begin{array}{c}\text { Indication for surgical revision* } \\
\text { (replacement, patient-specific implant) }\end{array}$
\end{tabular}

*A large gap or hole, or any instability, was considered indicative for surgical revision

Surgical revision was indicated if the bone flap resorption score was $\geq 3$. Thus, in accordance with the scoring described in Table 1, any palpable holes, instability, dislocation or collapse would indicate a need for surgical revision

\section{Bone flap resorption}

Resorption of the bone flap occurred in a considerable number of patients. Minor resorption as defined in Table 2 occurred in $20.7 \%$ (19/92) of patients and major resorption leading to surgical revision, and replacement with a patient-specific implant (PSI) occurred in $30.4 \%$ (28/92). Four patients (4.3\%) showed a relevant dislocation of the bone flap (Table 3). In $29.3 \%(27 / 92)$ of patients a PSI implantation was performed subsequently. The median time until identification of relevant bone resorption in clinical follow-up or imaging was 15.9 months (average 20.0, SD 15.1, range 1.0-47.7). Only 5 of the 28 cases were identified before 12 months after reimplantation.

\section{Statistical methods and power}

Only descriptive statistical methods were used. Ninety-two patients were included. The incidence of relevant bone resorption (grade 3 or higher) was $30.4 \%$, which led to a $95 \%$ confidence interval of 20.8 to $40 \%$.

Table 3 Complications after reimplatation of cryoconserved bone flaps

\begin{tabular}{lrc}
\hline Complication & $\mathrm{n}$ & $\%$ \\
\hline Infection of unknown origin & 0 & 0 \\
Infection after wound-healing disturbance & 1 & 1.1 \\
Hematoma & 3 & 3.3 \\
Hygroma & 12 & 13.0 \\
Hydrocephalus requiring shunt placement & 19 & 20.7 \\
Dislocation/collapse & 4 & 4.3 \\
Minor resorption (score 1-2) & 19 & 20.7 \\
Major resorption (score $\geq 3$ ) & 28 & 30.4 \\
\hline
\end{tabular}

The most commonly observed complications after reimplantation of cryoconserved bone flaps were resorption and hydrocephalus requiring shunt placement

\section{Discussion} Complications after reimplantation of cryoconserved bone
flaps

Due to the long time period required for performing surgery involving cryoprotected tissue, the number of publications dealing with the complications is rather small, especially in adult patients. Table 4 provides a summary of the literature, with an emphasis on the major complications reported. Age of patients, technique of bone flap preservation, time until reimplantation and underlying pathology differ considerably among these publications. Still, it is amazing how different the incidence of complications is, particularly the incidence of bone flap resorption. Grant et al. [6] found no bone resorption in children when the defect was smaller than $75 \mathrm{~cm}^{2}$. On the other hand, they found $60 \%$ relevant resorption in larger defects. Fragmentation of the bone flap and duration until reimplantation did not seem to influence the rate of resorption [6]. The next most important study is from Gruber et al., who reported $33 \%$ relevant resorption in a series of nine patients, with signs of minor bone resorption in all of the patients [7]. One very recent study by Schuss et al. [16] examined 254 patients retrospectively and provided a follow-up of more than 1 year for all cases. The patients were mainly adults, and the incidence of resorption was low (3.9\%) [16]. Unlike Grant et al., they found a significant influence of multifragmentation of the bone flap on the incidence of resorption. Schuss et al. confirmed that the timing of reimplantation was not relevant. Dünisch et al. [4] reported the largest series so far: 372 cases. Complications were well documented, and the mean followup was nearly 1 year. They found a very high rate of relevant bone resorptions of about $22 \%$ [4]. Unfortunately, for all the studies listed in Table 4 (except the present one), 'bone flap resorption' is not clearly defined. Schuss et al. define it as a prospective skull defect, instability or cosmetic deformation [16]. Dünisch et al. define a relevant resorption as necrosis of the tabula interna and externa [4]. The striking differences among the studies concerning this complication have their origin not only in the different ages of the patients, but also in the definitions of relevant resorption and the lengths of follow-up.

Reporting of bone resorption: illustrative case

One of the findings that was most surprising for us was that patients did not spontaneously report an occurrence of bone resorption. One typical example was a 17-year old boy who suffered a severe head trauma in a fistfight. A large subdural hematoma with major dislocation of the midline and consequent brain swelling led to decompressive hemicraniectomy. The bone was cryoconserved at $-80{ }^{\circ} \mathrm{C}$ until the reimplantation 6 weeks later. The patient was seen in the outpatient clinic 


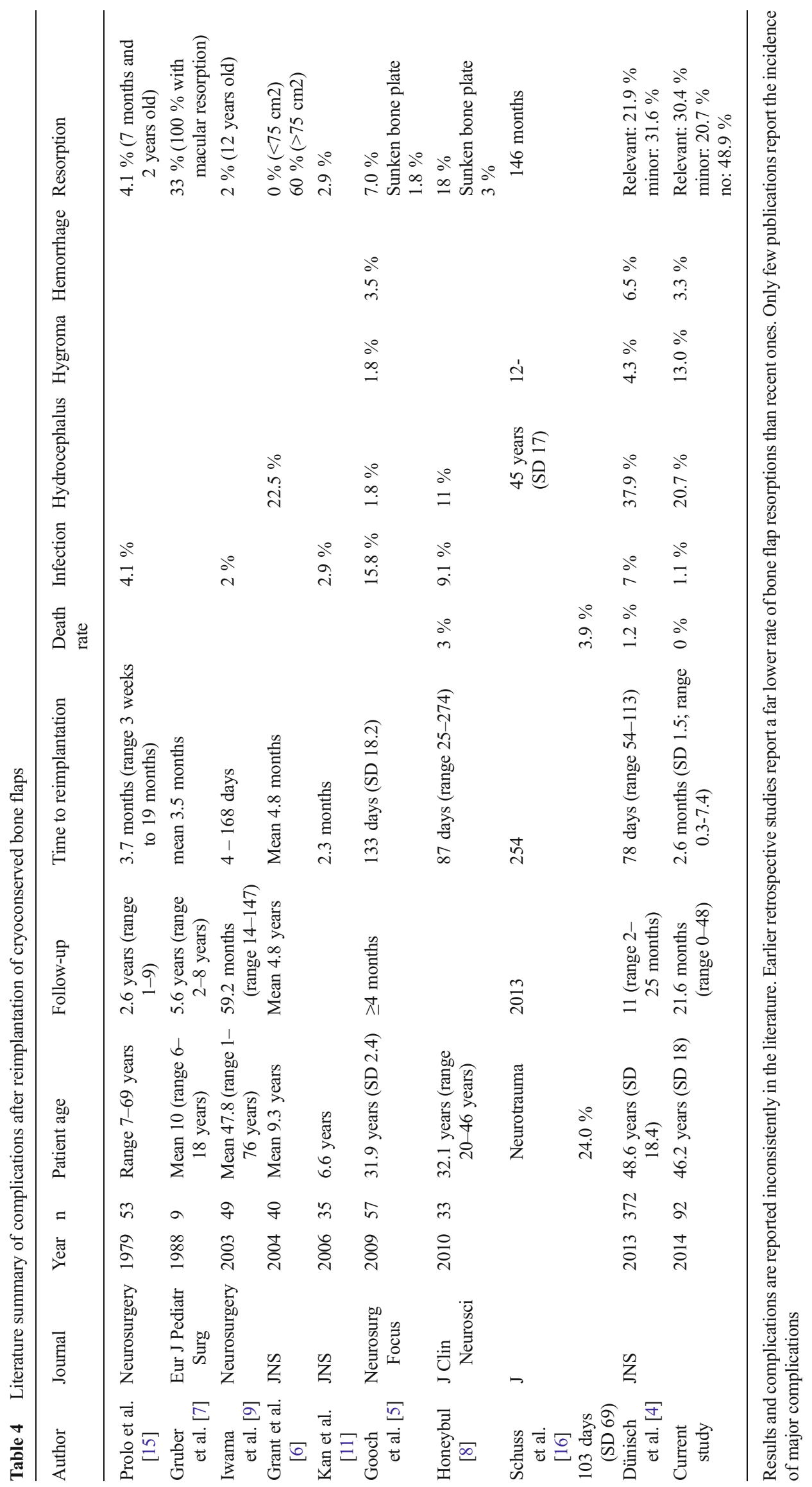


6 weeks after reimplantation, and the only neurological deficit was a minor gait disturbance. The bone flap fit perfectly, there were no signs of resorption, and the wound healing was good. The patient completed in-patient rehabilitation for 2 weeks and went back to work afterwards. In this young male the fast regrowth of curly black hair quickly covered the operated hemisphere. When the patient presented in our outpatient clinic for a late follow-up 6 months after the reimplantation, which was initiated by the hospital and not by the patient himself, he presented with major resorption, which was palpable as a $4 \mathrm{~cm}$ by $4 \mathrm{~cm}$ hole in the bone and retraction of the skin, but which was covered by his hair. The patient was aware of the skull defect but still did not realize that it was a problem.

Major resorptions were also identified in a similar manner in other patients. It appeared that patients with good recovery from the initial trauma/insult were satisfied with being able to lead a normal life again and did not want to complain about what they considered minor imperfections or perhaps were reluctant to undergo additional surgery. This lack of patient initiative may contribute to the low incidence of bone resorption reported in the literature, where the follow-up is generally short and clinical controls are initiated by the patient or the general practitioner.

\section{Definition of 'relevant' bone resorption}

The term 'bone resorption' must be defined in terms that will enable comparison between different centers and techniques currently in use or techniques that will be developed in the future. The reimplanted bone must at least fulfill the criteria given by Schuss et al.: to protect the brain and be cosmetically acceptable [16]. The best possible result would be fusion of the surrounding bone with no resorption (even histologically). Such fusion is unlikely, as histological and radiographic examinations by Prolo et al. showed signs of at least minor bone resorption in all cases [15]. A clear delineation between the indication for a surgical revision and a tolerable resorption is needed. We suggest the system described in Tables 1 and 2. This system can be applied to clinical parameters or to a CT scan. Instability or large holes in the bone flap indicate a need for immediate revision of the bone flap. Small holes and thinning of the bone flap may contribute, but are not by themselves sufficient to require revision of the bone flap.

The Bern experience: a high incidence of bone resorption

In our series of patients the incidence of bone flap resorption is rather high compared with the literature. It corresponds best with the findings of Dünisch et al., reporting $21 \%$ of patients with relevant bone resorptions [4]. We found relevant resorption (according to the definition described above) in $30.4 \%$ of cases and performed reoperation and replacement with a patient-specific implant (PSI) in 27 cases (29.3\%). Three patients with clear indications for bone flap replacement rejected the offer, despite the fact that in one case the dislocated bone flap was cosmetically unfavorable. We did not find a correlation with patient age, but the subgroup of patients under 20 was very small ( 8 patients, $37.5 \%$ ) and the study population was not powered for a subgroup analysis.

\section{Future aspects}

To learn the true incidence rate of the complications of this very common surgery is far from academic; it is highly relevant for clinical decision making and outcomes. Our own results, as well as publications by Gruber et al. [7] and Grant et al. [6], suggest that about one third of patients require replacement of the bone flap by allogenous material in an additional surgical approach. The rate of infection was rather low in our own series, but between 2 and $15.8 \%$ of patients reported in the literature (Table 4) experienced other complications such as hematomas and hygromas (13\% and $5 \%$ in our own series, respectively). If these results can be generalized, neurosurgeons must consider searching for alternatives to a surgical procedure with an approximately $40 \%$ overall complication rate. This question will never be answered satisfactorily on the basis of retrospective data, and there has never been a prospective study on this topic. Therefore, we suggest - and are currently planning - a multicenter prospective study. If our findings are supported by prospective results, the indication for reimplantation of autologous bone might be questioned in general. Alternatively, industrial patient-specific implants might be implanted from the very first. The higher costs for the implants might be put into perspective compared to the cost of repeated surgeries and the inconvenience for the patients. On the other hand, there are techniques available for using very inexpensive intraoperatively molded implants, for example, made from polymethylmethacrylate. Recent results showed that these individually molded implants are not inferior to commercial products with respect to cosmetic results and complication rates [17].

\section{Conclusions}

Bone flap resorption is a frequent complication after delayed reimplantation of cryoconserved bone flaps. The incidence of complications depends on the patient age and is more frequent in children than adults. There is also evidence for an influence of multisegmentation of the reimplanted flaps [16] and the size of the covered defect [6]. The overall incidence of major resorption reported in the literature is between 2 and $60 \%$. In our own series of 92 patients, the incidence was $30.4 \%(95 \%$ confidence interval: $20.8 \%$ to $40 \%$ ). To provide a clear 
definition of what we consider a major resorption, we present a simple scoring system that proved quite useful in our series. Some cases of bone flap resorption become evident only after a long follow-up. A future prospective study is planned to identify the optimal time point for clinical and radiological follow-up. Based on the present study, we expect the optimal follow-up time point to be at least 12 months after the reimplantation.

\section{Conflicts of interest None.}

\section{References}

1. Abbott KH (1953) Use of frozen cranial bone flaps for autogenous and homologous grafts in cranioplasty and spinal interbody fusion. $\mathrm{J}$ Neurosurg 10:380-388

2. Açikgöz B, Ozcan OE, Erbengi A, Bertan V, Ruacan S, Açikgöz HG (1986) Histopathologic and microdensitometric analysis of craniotomy bone flaps preserved between abdominal fat and muscle. Surg Neurol 26:557-561

3. Artico M, Ferrante L, Pastore FS, Ramundo EO, Cantarelli D, Scopelitti D, Iannetti G (2003) Bone autografting of the calvaria and craniofacial skeleton. Surg Neurol 60:71-79

4. Dünisch P, Walter J, Sakr Y, Kalff R, Waschke A, Ewald C (2013) Risk factors of aseptic bone resorption: a study after autologous bone flap reinsertion due to decompressive craniotomy. J Neurosurg 118: 1141-1147

5. Gooch MR, Gin GE, Kenning TJ, German JW (2009) Complications of cranioplasty following decompressive craniectomy: analysis of 62 cases. Neurosurg Focus 26(E9):1-7

6. Grant GA, Jolley M, Ellenbogen RG, Roberts TS, Gruss JR, Loeser JD (2004) Failure of autologous bone-assisted cranioplasty following decompressive craniectomy in children and adolescents. J Neurosurg Pediatr 100(2S):163-168

7. Gruber R, Peter R, Hora J (1988) The prognosis of cranioplasty following large craniectomy in children. Z Kinderchir 43:375-383

8. Honeybul S (2010) Complications of decompressive craniectomy for head injury. J Clin Neurosci 17:430-435

9. Iwama T, Yoshimura K, Keller E, Imhof H-G, Kahn N, Leblebicioglu-Konu D, Tanaka M, Valavanis A, Yonekawa Y (2003) Emergency craniotomy for intraparenchymal massive hematoma after embolization of supratentorial arteriovenous malformations. Neurosurgery 53:1251-1260

10. Iwama T, Yamada J, Imai S, Shinoda J, Funakoshi T, Sakai N (2003) The use of frozen autogenous bone flaps in delayed cranioplasty revisited. Neurosurgery 52:591-596

11. Kan P, Amini A, Hansen K, White GL, Brockmeyer DL, Walker ML, Kestle JR (2006) Outcomes after decompressive craniectomy for severe traumatic brain injury in children. J Neurosurg 105:337-342

12. Moreira-Gonzalez A, Jackson IT, Miyawaki T, Barakat K, DiNick V (2003) Clinical outcome in cranioplasty: critical review in long-term follow-up. J Craniofac Surg 14:144-153

13. Nakajima T, Someda K, Yamanouchi Y, Matsumura H (1977) Subcutaneous preservation of free skull bone flap taken out in decompressive craniectomy - a follow-up study. Neurol Surg Tokyo 5: $1329-1333$

14. Odom GL, Woodhall B, Wrenn FR (1952) The use of refrigerated autogenous bone flaps for cranioplasty. J Neurosurg 9:606-610

15. Prolo DJ, Durres KP, Mclaughlin WT, Christensen AH (1979) Autogenous skull cranioplasty: fresh and preserved (frozen), with consideration of the cellular response. Neurosurgery 4:18-29

16. Schuss P, Vatter H, Oszvald A, Marquardt G, Imöhl L, Güresir E (2013) Bone flap resorption: risk factors for the development of a long-term complication following cranioplasty after decompressive craniectomy. J Neurotrauma 30:91-95

17. Stieglitz LH, Gerber N, Schmid T, Mordasini P, Fichtner J, Fung C, Murek M, Weber S, Raabe A, Beck J (2014) Intraoperative fabrication of patient-specific moulded implants for skull reconstruction: single-centre experience of 28 cases. Acta Neurochir (Wien) 156: $793-803$ 